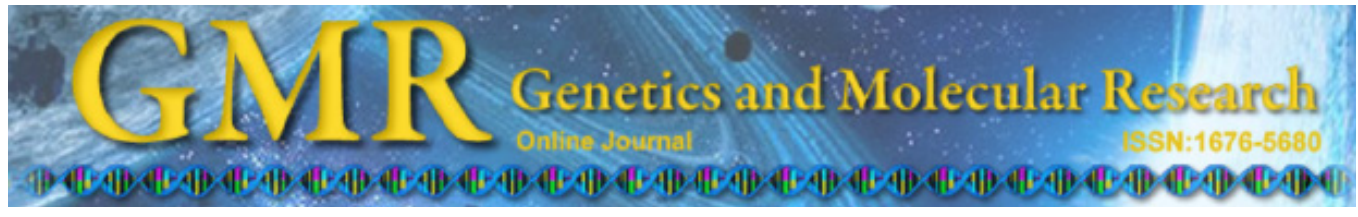

\title{
Zinc finger protein A20 overexpression inhibits monocyte homing and protects endothelial cells from injury induced by high glucose
}

C.L. Hou ${ }^{1 *}$, W. Zhang ${ }^{1 *}$, Y. Wei ${ }^{1}$, J.H. Mi ${ }^{1}$, L. Li ${ }^{1}$, Z.H. Zhou ${ }^{2}$, W. Zeng ${ }^{1}$ and D.J. Ying ${ }^{1}$

${ }^{1}$ Department of Anatomy, The Key Lab of Biomechanics, The Third Military Medical University, Chongqing, China ${ }^{2}$ Department of Neurology, Southwest Hospital, Third Military Medical University, Chongqing, China

*These authors contributed equally to this study. Corresponding author: D.J. Ying

E-mail: ydj96@yahoo.com.cn

Genet. Mol. Res. 10 (2): 1050-1059 (2011)

Received October 13, 2010

Accepted December 8, 2010

Published June 7, 2011

DOI 10.4238/vol10-2gmr1102

\begin{abstract}
Diabetes mellitus causes vascular lesions and may ultimately lead to atherosclerosis. One of the earliest steps in the development of atherosclerotic lesions is the adhesion of monocytes to endothelial cells of the vessel wall. It is currently unknown whether zinc finger protein A20 is able to protect endothelial cells from injury caused by high levels of glucose and monocyte homing. In our study, adhesion of monocytes to the vessel wall endothelium was detected by measuring the rolling velocity of monocytes along human umbilical vein endothelial cells (HUVECs). Activation of NF- $\mathrm{kB}$ was analyzed through Western blot. HUVEC apoptosis was monitored by TUNEL in situ end-labeling and flow cytometry. High glucose concentrations $(25 \mathrm{mM})$ stimulated monocytes, reducing the velocity at which they roll along HUVECs. Stimulation of monocytes with high levels of
\end{abstract}


glucose also induced HUVEC apoptosis. Overexpression of the zinc finger protein A20 inhibited monocyte recruitment, NF- $\mathrm{kB}$ activation, P-selectin expression, and HUVEC apoptosis induced by high glucose levels. We conclude that zinc finger protein A20 can protect HUVECs from injury induced by high levels of glucose and potentially could be used to develop treatments against diabetic vascular lesions.

Key words: A20 gene; HUVECs; High glucose; Apoptosis; Monocyte homing

\section{INTRODUCTION}

Diabetes mellitus is a metabolic disease caused by insulin deficiency and/or a disturbance in insulin levels. The main characteristic of diabetes mellitus is chronic hyperglycemia. Previous studies have shown that hyperglycemia can promote the adhesion of monocytes to vessel wall endothelia, change vascular endothelial structure and function, and ultimately lead to atherosclerosis. Therefore, hyperglycemia is considered to be primary cause of diabetic vascular lesions.

A20 was originally identified as a tumor necrosis factor (TNF)-inducible gene in human umbilical vein endothelial cells (HUVECs). Recent studies have shown that A20 is essential for the prevention of spontaneous innate immune cell-mediated inflammation and tissue destruction. It can inhibit the activation of a number of reporters in endothelial cells, including vascular cell adhesion molecule 1 (VCAM-1), E-selectin, interleukin-8, and tissue factor. It has also been reported that A20 can inhibit the induction of endothelial cell apoptosis mediated by factors such as lipopolysaccharide (LPS) and TNF (Zhu et al., 2004; Patel et al., 2006; Zeng et al., 2009). However, it is unknown whether the A20 gene is expressed in endothelial cells that have been stimulated by exposure to high levels of glucose. It is also unknown whether A20 can protect endothelial cells from injuries mediated by high concentrations of glucose. Recent research indicates that hyperglycemia induced endothelial cell injury is related to nuclear factor kappa B (NF-kB) (Yang et al., 2008). One of the molecular mechanisms is that A20 can block NF- $\mathrm{kB}$ signaling (Wang et al., 2007; Lutz et al., 2008). In this study, we investigated whether the effect of A20 was related to NF-kB signaling pathway under high glucose condition.

\section{MATERIAL AND METHODS}

\section{Isolation and culture of HUVECs}

HUVECs were prepared and cultured as described previously (Wang et al., 2007). The cells were used in experiments after they had undergone two or three passages. The cells were exposed to medium containing $25 \mathrm{mM}$ D-glucose (high) or $5 \mathrm{mM} \mathrm{D}$-glucose plus $20 \mathrm{mM}$ mannitol as an osmotic control (McGinn et al., 2003).

\section{Analysis of A20 expression in endothelial cells}

A20 expression was detected in the transfected HUVECs using immunofluorescence. 
In addition, total mRNA was isolated from the HUVECs, and reverse transcriptase-polymerase chain reaction (RT-PCR) was used to analyze A20 expression. A one-step RT-PCR kit (Promega Corp., USA) was used for RT-PCR analysis with the following primers for A20: sense, 5'-ATGGCTGAACAAGTCCTTCC-3', and antisense, 5'-TTAGCCATACATCT GCTTGAA-3' (synthesized by TaKaRa Biotech Co., Ltd., Dalian, China). Immunofluorescence and RT-PCR analyses were performed as described previously (Zhu et al., 2004).

\section{Transfection protocol}

When the HUVECs reached $75 \%$ confluence in $24-w e l l$ plates, they were transfected with pcDNA3.1EHA20 using the TransFast ${ }^{\mathrm{TM}}$ Transfection Reagent according to manufacturer instructions. pcDNA3.1 was transfected into HUVECs as the control.

\section{Monocyte rolling assay}

Human monocytes were obtained using a modified Recalde method (Fogelman et al., 1988). Monocytes were collected by venipuncture into Vacutainer tubes that contained $10 \mathrm{U} /$ $\mathrm{mL}$ heparin. For the rolling assays, monocytes were drawn through a parallel flow chamber at controlled flow rates using a Nano Syringe Pump (KDS 210 pump, Focus, Hong Kong) after the designated experimental flow regimen had been completed. The rolling velocity of the monocytes along HUVECs was recorded using a camera (Basler, Germany) (Mohan et al., 1999).

\section{NF- $\mathrm{B}$ activation}

Nuclear protein was extracted and NF- $\mathrm{KB}$ activation was analyzed through Western blot after HUVECs being cultured in high glucose $(25 \mathrm{mM})$ medium for $12 \mathrm{~h}$. The Protein Extraction Kit was purchased from Shanghai Bioengineering Corp. The detailed procedure was performed according to the manual and $\mathrm{DAB}$ coloration was performed.

\section{P-selectin expression}

P-selectin expression in the HUVECs was detected using immunofluorescence after the HUVECs had been cultured in high glucose medium for $12 \mathrm{~h}$. The primary antibody was a rabbit anti-P-selectin antibody (1:200), and the secondary antibody was a Rhodamine (TRITC)-conjugated AffiniPure goat anti-rabbit IgG. P-selectin immunofluorescence was analyzed and quantified by confocal fluorescence microscopy.

\section{In situ terminal deoxynucleotidyl transferase biotin-dUTP nick-end labeling (TUNEL) analysis of apoptosis}

HUVECs were harvested after stimulation by high glucose for $72 \mathrm{~h}$. They were then fixed using $4 \%$ paraformaldehyde for $30 \mathrm{~min}$ at $4{ }^{\circ} \mathrm{C}$, then incubated for $20 \mathrm{~min}$ in $3 \% \mathrm{H}_{2} \mathrm{O}_{2} /$ methanol at room temperature. Triton X-100 (0.1\%, diluted with $0.1 \%$ natrium citricum) was then added to the cells, and they were incubated on ice for $2 \mathrm{~min}$. Fifty microliters TUNEL reagent was then added to the cells and they were incubated for $1 \mathrm{~h}$ at $37^{\circ} \mathrm{C}$. The cells were 
washed with PBS, and then stained with a fluorescently labeled antibody against endotheliocyte VIII factor, SABC-Cy3. The number of cells was counted using fluorescence microscopy.

\section{Flow cytometric analysis of apoptosis}

In order to further analyze the apoptosis induced by high glucose, the HUVECs were stimulated with high glucose for $72 \mathrm{~h}$. The apoptotic cells were labeled with an Annexin VFITC Kit (Jingmei Biotech Co., Ltd., Beijing, China), and then analyzed by flow cytometry.

\section{Data analysis}

The data were analyzed using SPSS13.0 and are reported as means \pm SD. All statistical analyses were conducted using the alternate Welch's method. A P value $<0.05$ was considered to be significant.

\section{RESULTS}

\section{Analysis of A20 mRNA expression by RT-PCR}

We also examined A20 expression in HUVECs using RT-PCR in order to determine whether A20 expression could be induced by high glucose. Little to no A20 mRNA was detected in HUVECs cultured under normal conditions. A20 expression was induced by high glucose, and A20 mRNA could rapidly increase within as little as $2 \mathrm{~h}$ in our RT-PCR analyses (Figure 1), but little to no A20 mRNA was detected after high glucose stimulation for $24 \mathrm{~h}$. These results indicate that A20 is an early response gene in HUVECs activated by high concentrations of glucose.

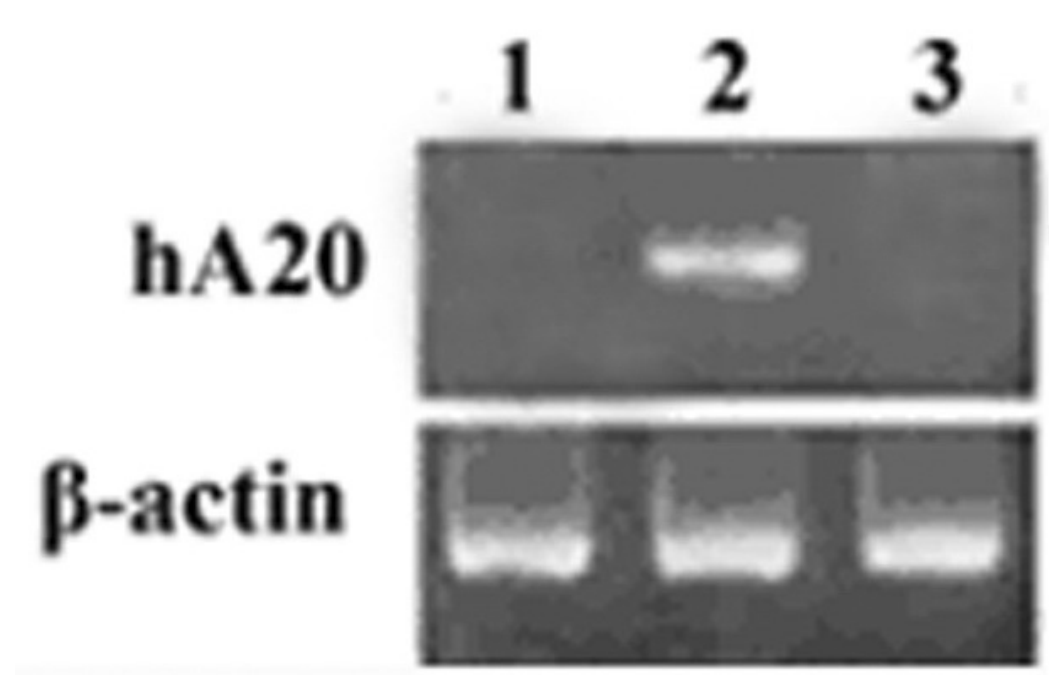

Figure 1. A20 expression was analyzed by RT-PCR. Lane $1=$ HUVECs were cultured in normal medium; lane 2 $=$ HUVECs were stimulated by high glucose for $2 \mathrm{~h}$; lane $3=$ HUVECs were stimulated by high glucose for $24 \mathrm{~h}$. 


\section{Stable transfection of HUVECs with A20}

The pcDNA3.1EHA20 plasmid was stably integrated into HUVECs, thus providing the opportunity to study the effect of A20 inhibition upon endogenous gene expression in a regulated manner. Plasmid transfer of A20 using the TransFast ${ }^{\mathrm{TM}}$ Transfection Reagent with G418 selection led to A20 expression levels of over 95\% in the HUVECs. Immunofluorescence analyses of HUVECs transfected with pcDNA3.1EHA20 revealed a high level of A20 expression in almost all cells (Figure 2). However, there was no observable fluorescence in the pcDNA3.1-transfected control endothelial cells. In addition, we observed that A20 was mainly present in the cytoplasm, which is consistent with its endogenous pattern of expression. The first antibody is mouse anti-E-tag MoAb; the anti-E-tag MoAb used in these experiments did not cross-react with human A20, because only the exogenous plasmid pcDNA3.1EHA20 includes an E-tag. The second antibody is Rhodamine (TRITC)-conjugated AffiniPure Goat Anti-Mouse IgG.
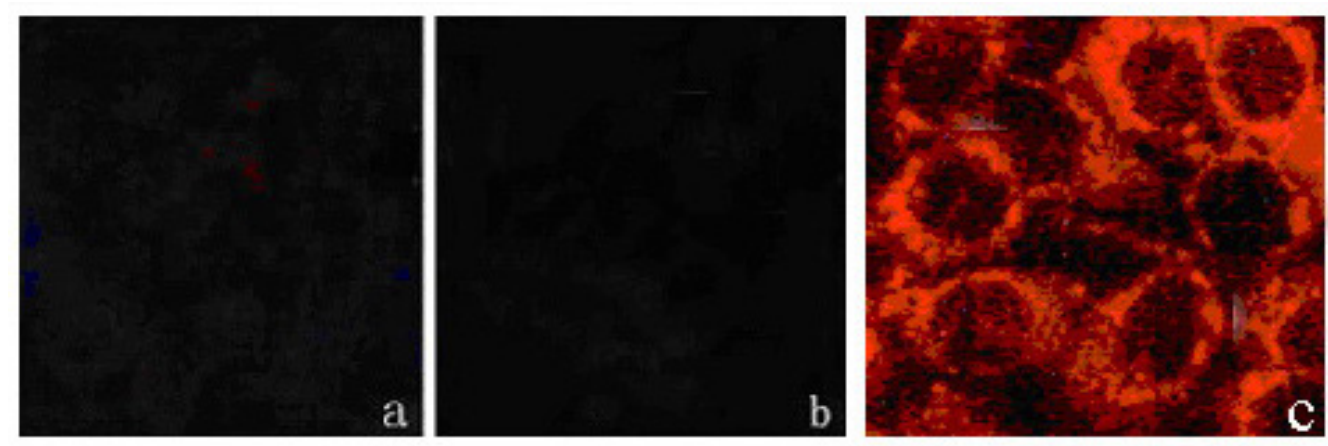

Figure 2. The expression of hA20 is checked by laser scanning confocal microscopy. a. HUVECs. b. HUVECs with plasmid pcDNA3.1. c. HUVECs with plasmid pcDNA3.1EHA20.

\section{Monocyte homing}

The reduced velocity rolling of monocytes along the endothelial surface plays an important role in the early stages of inflammation. In the control group (cells were cultured in normal medium), the rolling rate of monocytes was $0.6089 \pm 0.051 \mathrm{~mm} / \mathrm{s}$; in the pcDNA3.1transfected group (cells were cultured in high glucose medium), the rolling rate was 0.4551 $\pm 0.052 \mathrm{~mm} / \mathrm{s}$, and in the pcDNA3.1EHA20-transfected group (cells were cultured in high glucose medium), the rolling rate was $0.5842 \pm 0.023 \mathrm{~mm} / \mathrm{s}$ (Figure 3). There was a statistically significant difference in the rolling rates between the control group of cells and the pcDNA3.1-transfected group $(\mathrm{P}<0.01)$, indicating that high levels of glucose could increase the adhesion between monocytes and HUVECs. The difference between the rolling rates in the pcDNA3.1EHA20 and pcDNA3.1 groups was also significant $(\mathrm{P}<0.01)$; however, there was no significant difference between the rolling rates of the pcDNA3.1EHA20 and the control group $(\mathrm{P}>0.05)$, indicating that expression of A20 could prevent the reduction in the velocity of monocyte rolling mediated by high levels of glucose. These results imply that A20 plays an important role in the prevention of inflammation. 


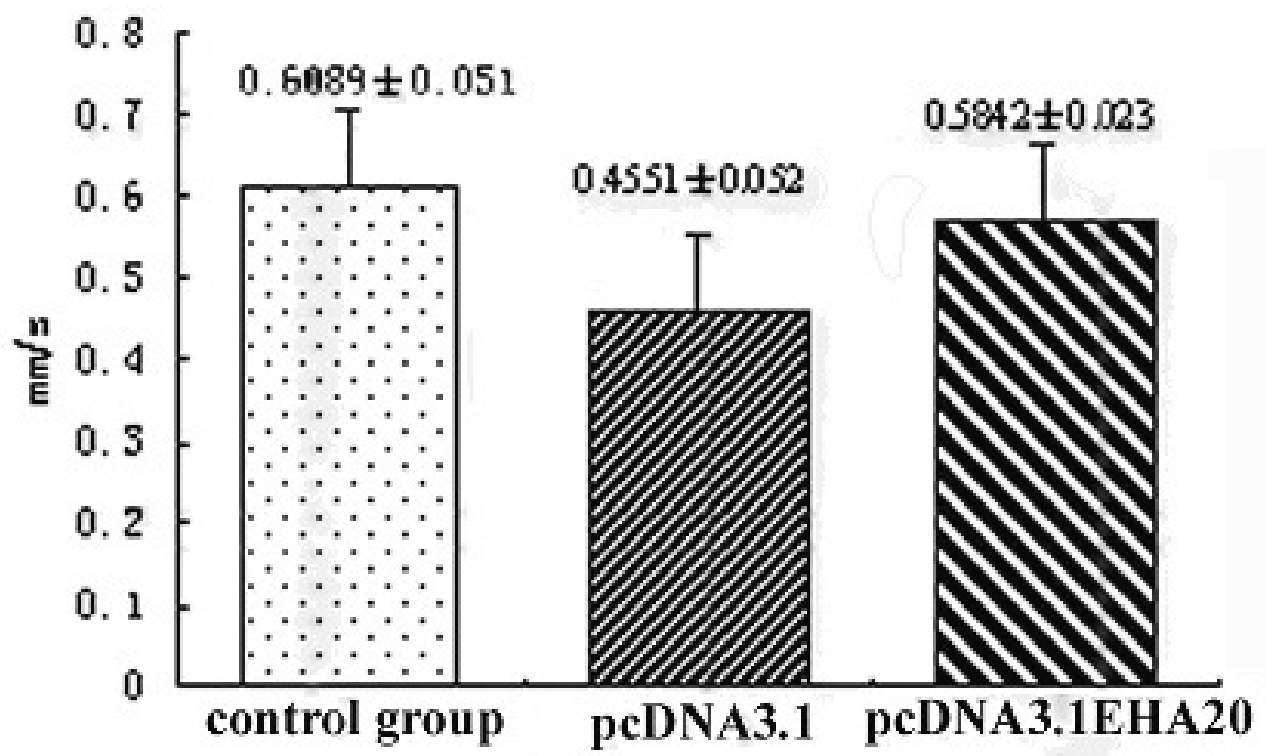

Figure 3. Monocytes rolling rate along HUVECs. In the control group, HUVECs were cultured in normal medium. In the PcDNA3.1 and pcDNA3.1EHA20 groups, HUVECs were cultured in high glucose medium.

\section{NF- $\mathrm{B}$ activation}

We found that NF-кB activation was greatly increased by high glucose in the pcDNA3.1-transfected group, but was inhibited in the pcDNA3.1EHA20-transfected group (Figure 4).

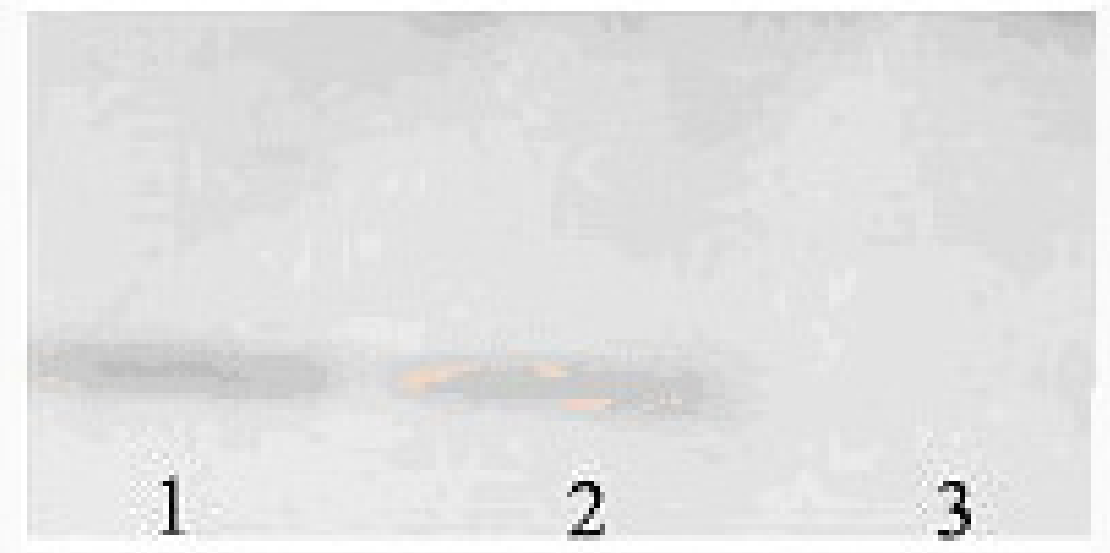

Figure 4. Western blot analysis of NF-kB activation when HUVECs was stimulated by high glucose for $12 \mathrm{~h}$. Lane 1 = HUVECs; lane 2 = HUVECs with plasmid pcDNA3.1; lane 3 = HUVECs with plasmid pcDNA3.1EHA20. 


\section{P-selectin expression}

The results of the immunofluorescence analyses demonstrated that treatment of HUVECs with high levels of glucose induced the expression of P-selectin. However, expression of A20 significantly inhibited the expression of P-selectin in the HUVECs (Figure 5), and the percentage inhibition was greater than $80-90 \%$.

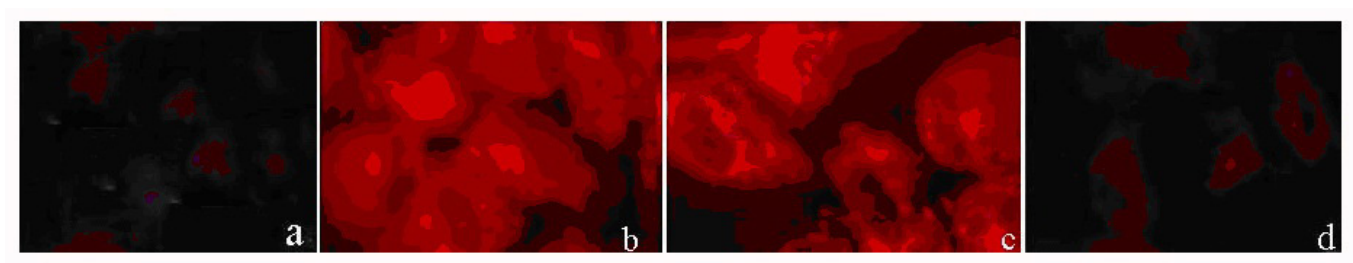

Figure 5. The expression of P-selectin in HUVECs was stimulated by high glucose for $12 \mathrm{~h}(\mathrm{~b}, \mathrm{c}$, and d) at $1000 \mathrm{X}$. a. Blank control (cultured in normal medium). b. HUVECs. c. HUVECs with plasmid pcDNA.1. d. HUVECs with plasmid pcDNA3.1EHA20.

\section{Overexpression of A20 inhibits HUVECs apoptosis induced by high glucose}

It has previously been reported that A20 is an early response gene and can protect cells against cytokine-mediated cytotoxicity. Other studies have shown that hyperglycemia can damage endothelial cells and induce their apoptosis. Therefore, we tested whether A20 could protect HUVECs against injury induced by high glucose (Figure 6; Table 1; Figure 7).
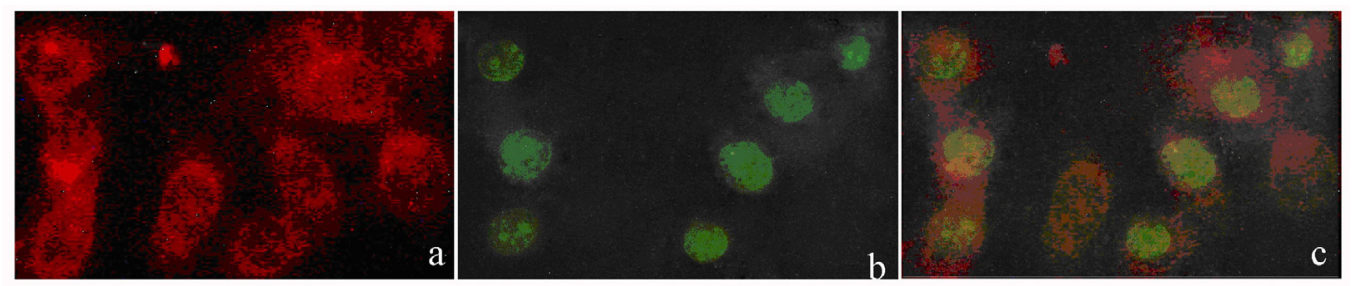

Figure 6. The apoptosis of HUVECs was determined by fluorescence microscopy at 400X. a. HUVECs were determined by von Willebrand factor. b. The apoptosis of HUVECs in the same visual field was counted by fluorescence microscopy. c. $a+b$.

Table 1. The apoptosis of human umbilical vein endothelial cells (HUVECs) was counted through a fluorescence microscope.

\begin{tabular}{lccc}
\hline HUVECs & Blank control & pcDNA3.1 & pcDNA3.1EHA20 \\
\hline In high level glucose medium & $28 \pm 2 \% *$ & $29 \pm 1 \% *$ & $11 \pm 1 \% * *$ \\
In normal medium & $4 \pm 1 \%$ & $6 \pm 2 \%$ & $6 \pm 1 \%$ \\
\hline
\end{tabular}

$* \mathrm{P}<0.01$ compared with HUVECs in normal medium. ${ }^{* *} \mathrm{P}<0.01$ compared with the pcDNA3.1-transfected group or the control group cultured in high glucose medium. 

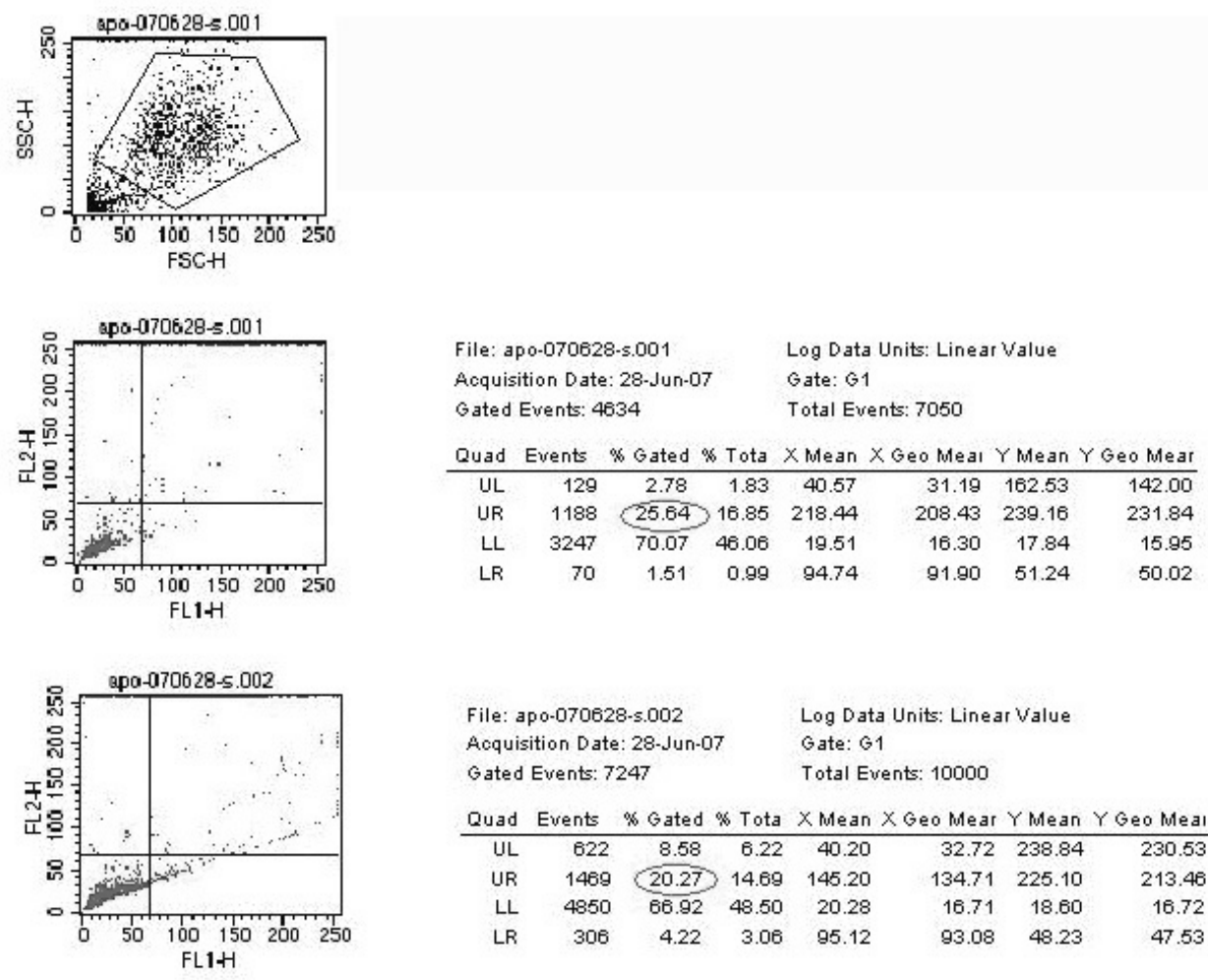

\begin{tabular}{|c|c|c|c|c|c|c|c|}
\hline & \multicolumn{4}{|c|}{ Log Data Units: Linear Value } \\
\hline \multicolumn{4}{|c|}{ Acquisition Date: $28-J u n-07$} & \multicolumn{4}{|c|}{ Gate: $\mathrm{G1}$} \\
\hline \multicolumn{4}{|c|}{ Gated Events: 7247} & \multicolumn{4}{|c|}{ Total Events: 10000} \\
\hline Quad & Events & \& Gated & \& Tota & $X$ Mean & X Geo Mear & YMean & YGeo Meal \\
\hline UL & 622 & 8.58 & 6.22 & 40.20 & 32.72 & 238.84 & 230.53 \\
\hline UR & 1469 & 20.27 & 14.69 & 145.20 & 134.71 & 225.10 & 213.46 \\
\hline LL & 4850 & $6 \overline{6.92}$ & 48.50 & 20.28 & 16.71 & 18.60 & 16.72 \\
\hline LR & 306 & 4.22 & 3.06 & 95.12 & 93.08 & 48.23 & 47.53 \\
\hline
\end{tabular}

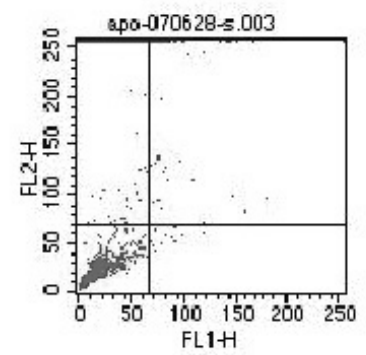

\begin{tabular}{|c|c|c|c|c|c|c|c|}
\hline \multicolumn{4}{|c|}{ File: apo-070628-s.003 } & \multicolumn{4}{|c|}{ Log Data Units: Linear Value } \\
\hline \multicolumn{4}{|c|}{ Acquisition Date: 28 -Jun-07 } & \multicolumn{4}{|l|}{ Gate: $G 1$} \\
\hline \multicolumn{4}{|c|}{ Gated Events: 7472} & \multicolumn{4}{|c|}{ Total Events: 10000} \\
\hline Quad & Events & \& Gated & \& Tota & $x$ Mean & X Geo Meal & YMean & YGeo Mear \\
\hline UL & 1924 & 25.75 & 19.24 & 26.67 & 17.65 & 243.49 & 237.27 \\
\hline UR & 260 & 3.48 & 2.60 & 105.48 & 98.49 & 208.56 & 192.85 \\
\hline LL & 5217 & 69.82 & 52.17 & 16.61 & 13.71 & 18.96 & 16.77 \\
\hline LR & 71 & 0.95 & 0.71 & 86.89 & 85.66 & 53.83 & 53.31 \\
\hline
\end{tabular}

Figure 7. The apoptosis was detected through flow cytometric analysis. 001. HUVECs without any plasmid. 002. HUVECs with plasmid pcDNA3.1. 003. HUVECs with plasmid pcDNA3.1EHA20.

The results from the TUNEL and flow cytometry analyses indicated that high levels of glucose could induce significant apoptosis in HUVECs. In the blank control, the percentage of apoptotic cells was $28 \pm 2 \%$, and in cells transfected with pcDNA3.1, this percentage was $29 \pm$ $1 \%$. The difference between the levels of apoptosis in these two groups was not significant ( $\mathrm{P}$ $>0.05$ ), and a similar result was obtained by flow cytometry (25.64 and 20.27\%). In contrast, the percentage of apoptotic cells in the HUVECs transfected with pcDNA3.1EHA20 was much lower (11 \pm 1 and $3.48 \%$, respectively) than that in the cells transfected with pcDNA3.1 and the 
blank control, and there was a significant difference $(\mathrm{P}<0.01)$. Therefore, these experiments demonstrated that A20 could protect HUVECs from the apoptosis induced by high glucose.

\section{DISCUSSION}

A20 is an early response gene in endothelial cells and can protect against the inflammation and apoptosis induced by LPS, CD40 ligation, the LMP1 protein of EBV, and the Tax protein of HIV. However, it is unknown whether A20 can maintain its protective function in HUVECs stimulated by treatment with high concentrations of glucose. In order to investigate the protective role of A20 under hyperglycemic conditions, a plasmid encoding for the A20 cDNA was transfected into HUVECs using the TransFast ${ }^{\mathrm{TM}}$ Transfection Reagent, and the cells expressing A20 were selected using G418.

There is evidence to suggest that endothelial dysfunction may be involved in the pathogenesis of diabetic macro- and microangiopathy (La Fontaine et al., 2006). Recent studies have implicated hyperglycemia in the pathogenesis of macro- and microvascular disease in diabetes mellitus. Exposure of the endothelium to hyperglycemic conditions can lead to endothelial dysfunction and apoptosis. Most investigators believe that high glucose concentrations can induce oxidative stress and apoptosis in endothelial cells though NF- $\mathrm{kB}$ signaling (Romero et al., 2008). A20 is an important protective gene for endothelial cells. One of the mechanisms is that A20 can block NF- $\kappa B$ signaling, while NF- $\kappa B$ signaling is very important in endothelial injury and apoptosis induced by hyperglycemic conditions (Zhu et al., 2004). We suppose that A20 protects endothelial cells against apoptosis and monocyte adhesion induced by high glucose throuth NF- $\mathrm{kB}$ blocking.

Our results indicate that high glucose concentrations can induce HUVEC injury and apoptosis, while at the same time increasing monocyte adhension to HUVECs. This may be an important reason why vascular lesion incidence is very high in the diabetics. A20 can inhibit NF- $\kappa \mathrm{B}$ activation. A20 overexpression can protect HUVECs against apoptosis induced by high glucose. A20 leads to a decrease in the percentage of apoptotic cells, and there is a significant difference in the number of apoptotic cells when compared with the control HUVECs transfected with pcDNA3.1.

P-selectin belongs to the selectin family of adhesion molecules, and is important for the initiation and maintenance of the inflammatory reaction. Therefore, P-selectin is a good marker of endothelial cell inflammation. It plays an important role in endothelial cell activation, leukocyte adhesion and leukocyte infiltration. Our results show that there is no P-selectin expression in HUVECs cultured under normal conditions, and there is no storage in the endochylema. However, when HUVECs are stimulated by high concentrations of glucose, Pselectin expression sharply increases. This indicates that high levels of glucose can activate endothelial cells. A20 overexpression, however, can inhibit $>80 \%$ of the P-selectin expression induced by treatment with high levels of glucose when compared with the control group.

Previous reports have clarified that high glucose can induce NF- $\kappa B$ expression. NF$\kappa \mathrm{B}$ is a nuclear factor functioning in a number of transcription regulations. It can activate the expression of more than 150 kinds of factors, and is the focal point of multiple signal transduction pathways. P-selectin belongs to the adhesion molecule family and is also one of the important downstream effector molecules of NF- $\mathrm{kB}$. It has been reported that in order for monocytes to enter a tissue, they must first undergo a cascade of events during the early stages 
of inflammation. These events consist of monocyte rolling, adhesion and migration. These reactions are regulated by adhesion molecules expressed in monocytes and on the surface of endothelial cells, in which P-selectin is one of the important regulatory molecules. NF- $\mathrm{kB}$ activation can promote the expression of P-selectin in endothelial cells. Binding of P-selectin to its ligand (monocyte expression of PSGL-1) promotes monocyte adhesion, and high glucose induces apoptosis of endothelial cells, exposing the subcutaneous tissue, which speeds up monocyte adhesion and migration and thus leads to inflammation. Our results indicated that high glucose can increase P-selectin expression on the endothelial cell surface and decrease the velocity of monocyte rolling along HUVECs. This provides the monocytes with the opportunity to adhere to HUVECs. By inhibiting NF- $\kappa B$ activation, A20 may reduce P-selectin expression and inhibit monocyte adhesion, demonstrating that A20 plays an important role in inhibiting the initiation of the inflammatory reaction. Meanwhile, A20 can protect endothelial cells against apoptosis induced by high glucose, leading to the protection of vascular endothelium.

Therefore, we have shown that A20 is a potent protector of the apoptosis induced by high levels of glucose, suggesting that, in the future, it may play an important role in the treatment of diabetic vascular lesions.

\section{ACKNOWLEDGMENTS}

Research supported by the National Science Foundation of China (\#30700771) and the Natural Science Foundation of Chongqing (\#CSTC, 2010BB5176).

\section{REFERENCES}

Fogelman AM, Elahi F, Sykes K, Van Lenten BJ, et al. (1988). Modification of the Recalde method for the isolation of human monocytes. J. Lipid Res. 29: 1243-1247.

La Fontaine J, Harkless LB, Davis CE, Allen MA, et al. (2006). Current concepts in diabetic microvascular dysfunction. J. Am. Podiatr. Med. Assoc. 96: 245-252.

Lutz J, Luong Le A, Strobl M, Deng M, et al. (2008). The A20 gene protects kidneys from ischaemia/reperfusion injury by suppressing pro-inflammatory activation. J. Mol. Med. 86: 1329-1339.

McGinn S, Saad S, Poronnik P and Pollock CA (2003). High glucose-mediated effects on endothelial cell proliferation occur via p38 MAP kinase. Am. J. Physiol. Endocrinol. Metab. 285: E708-E717.

Mohan S, Mohan N, Valente AJ and Sprague EA (1999). Regulation of low shear flow-induced HAEC VCAM-1 expression and monocyte adhesion. Am. J. Physiol. 276: C1100-C1107.

Patel VI, Daniel S, Longo CR, Shrikhande GV, et al. (2006). A20, a modulator of smooth muscle cell proliferation and apoptosis, prevents and induces regression of neointimal hyperplasia. FASEB J. 20: 1418-1430.

Romero MJ, Platt DH, Tawfik HE, Labazi M, et al. (2008). Diabetes-induced coronary vascular dysfunction involves increased arginase activity. Circ. Res. 102: 95-102.

Wang AB, Li HL, Zhang R, She ZG, et al. (2007). A20 attenuates vascular smooth muscle cell proliferation and migration through blocking PI3k/Akt singling in vitro and in vivo. J. Biomed. Sci. 14: 357-371.

Yang WS, Seo JW, Han NJ, Choi J, et al. (2008). High glucose-induced NF-kappaB activation occurs via tyrosine phosphorylation of IkappaBalpha in human glomerular endothelial cells: involvement of Syk tyrosine kinase. Am. J. Physiol. Renal Physiol. 294: F1065-F1075.

Zeng W, Li L, Yuan W, Wei Y, et al. (2009). A20 overexpression inhibits low shear flow-induced CD14-positive monocyte recruitment to endothelial cells. Biorheology 46: 21-30.

Zhu CH, Ying DJ, Mi JH, Zhang W, et al. (2004). The zinc finger protein A20 protects endothelial cells from burns serum injury. Burns 30: 127-133. 